\title{
Stachydrine protects eNOS uncoupling and ameliorates endothelial dysfunction induced by homocysteine
}

\author{
Xinya Xie ${ }^{1}$, Zihui Zhang ${ }^{1}$, Xinfeng Wang ${ }^{1}$, Zhenyu Luo ${ }^{1}$, Baochang Lai ${ }^{1}$, Lei Xiao ${ }^{1 *}$ and Nanping Wang ${ }^{2 *}$
}

\begin{abstract}
Background: Hyperhomocysteinemia (HHcy) is an independent risk factor for cardiovascular diseases (CVDs). Stachydrine (STA) is an active component in Chinese motherwort Leonurus heterophyllus sweet, which has been widely used for gynecological and cardiovascular disorders. This study is aimed to examine the effects of STA on homocysteine (Hcy)-induced endothelial dysfunction.
\end{abstract}

Methods: The effects of STA on vascular relaxation in rat thoracic aortas (TA), mesenteric arteries (MA) and renal arteries (RA) were measured by using Multi Myograph System. The levels of nitric oxide (NO), tetrahydrobiopterin (BH4) and guanosine 3', 5' cyclic monophosphate (cGMP) were determined. Endothelial nitric oxide synthase (eNOS) dimers and monomers were assayed by using Western blotting. GTP cyclohydrolase 1 (GTPCH1) and dihydrofolate reductase (DHFR) expressions were measured by using quantitative reverse transcriptase-PCR (qRT-PCR) and Western blotting.

Results: STA effectively blocked Hcy-induced impairment of endothelium-dependent vasorelaxation in rat TA, MA and RA. STA-elicited arterial relaxations were reduced by NOS inhibitor NG-nitro-L-arginine methyl ester (L-NAME) or the NO-sensitive guanylyl cyclase inhibitor $1 \mathrm{H}$ - $[1,2,4]$ Oxadiazolo[4,3-a]quinoxalin-1-one (ODQ), but not by inducible iNOS inhibitor $1400 \mathrm{~W}$ nor the nonselective COX inhibitor indomethacin. Hcy caused eNOS uncoupling and decreases in NO, CGMP and BH4, which were attenuated by STA. Moreover, STA prevented decreases of GTPCH1 and DHFR levels in Hcy-treated BAECs.

Conclusion: We demonstrated that STA effectively reversed the Hcy-induced endothelial dysfunction and prevented eNOS uncoupling by increasing the expression of GTPCH1 and DHFR. These results revealed a novel mechanism by which STA exerts its beneficial vascular effects.

Keywords: Stachydrine, GTPCH1, DHFR, eNOS uncoupling, Vasorelaxation

\section{Background}

Stachydrine (STA) is a major constituent of Chinese motherwort Leonurus heterophyllus sweet, which has been used in traditional medicine to promote blood circulation and dispel blood stasis (Yin et al., 2010). STA is also highly present in L. japonicus, L. cardiaca fruits, Leonotis leonurus (Kuchta et al., 2013) as well as in citrus fruits (Servillo et al., 2013). Several studies have shown that STA has protective effects on vascular endothelial cells (ECs).

\footnotetext{
* Correspondence: xiaolei0122@xjtu.edu.cn; nanpingwang2003@yahoo.com ${ }^{1}$ Cardiovascular Research Center, School of Basic Medical Sciences, Xi'an Jiaotong University, Xi'an 710061, China

${ }^{2}$ The Advanced Institute for Medical Sciences, Dalian Medical University, Dalian 116044, China
}

STA protected endothelial against the injury induced by anoxia-reoxygenation (Yin et al., 2010). STA effectively reduced lipopolysaccharide (LPS)-induced endothelial inflammatory response via the inhibition of interleukin (IL-10) and thromboxane B $2\left(\mathrm{TXB}_{2}\right)$ secretion (Hu et al., 2015; Hu et al., 2012). STA inhibited the deleterious effect of high glucose on ECs and acted through the modulation of SIRT1 pathway (Servillo et al., 2013). However, little is known about STA on vascular relaxation, a common feature of endothelial function.

Hyperhomocysteinemia (HHcy) is an independent risk factor for various cardiovascular diseases (CVDs) (Karolczak et al., 2013; Baggott \& Tamura, 2015). 
Homocysteine (Hcy) exerts its adverse effect on endothelial function by increasing oxidative stress and inhibiting the activity of endothelial nitric oxide synthase (eNOS) and decreasing nitric oxide (NO) production (Cheng et al., 2015). A critical determinant of eNOS activity is its cofactor tetrahydrobiopterin ( $\mathrm{BH} 4)$. $\mathrm{BH} 4$ can be formed either by a de novo biosynthetic pathway using the ratelimiting enzyme GTP-cyclohydrolase I (GTPCH1) or a salvage pathway from sepiapterin, which is dependent on dihydrofolate reductase (DHFR) (Hussein et al., 2015; Haruki et al., 2016). An inadequate level of BH4 makes eNOS no longer coupled to L-arginine oxidation (eNOS uncoupling) and results in the production of reactive oxygen species (ROS) rather than $\mathrm{NO}$, thereby leading to vascular endothelial dysfunction (Takimoto et al., 2005).

In this study, we investigated the effects of STA on the Hcy-induced endothelial dysfunction and with the emphasis on its role in eNOS uncoupling and the underlying mechanism.

\section{Methods \\ Reagents}

STA was obtained from Cayman Chemical (Ann Arbor, MI, USA). Dimethyl sulfoxide (DMSO), 3-[4,5-dimethylthiazol-2-yl]-2,5-diphenyl-tetrazolium bromide (MTT), acetylcholine (ACh), indomethacin, NG-nitro-L-arginine methyl ester (L-NAME), 1H- (Yin et al., 2010; Kuchta et al., 2013; Hu et al., 2015) Oxadiazolo[4,3-a]quinoxalin-1one (ODQ), $1400 \mathrm{~W}, \mathrm{Hcy}$, angiotensin II (Ang II), palmitic acid (PA) and rabbit polyclonal antibody to GTPCH1 were purchased from Sigma-Aldrich (St. Louis, MO, USA). The antibody against eNOS was purchased from Cell Signaling Technology (Danvers, MA, USA). Mouse monoclonal antibodies to DHFR and $\beta$-actin, HRP-conjugated anti-rabbit and anti-mouse IgG polyclonal antibodies were procured from Santa Cruz Biotechnology (Santa Cruz, CA, USA). ELISA kit for cGMP was obtained from R\&D Systems Inc. (R\&D Systems, MN, USA). BH4 ELISA kit was from MyBioSource Inc. (San Diego, CA, USA). Dulbecco's modified Eagle medium (DMEM), fetal bovine serum (FBS) and 4-amino-5-methylamino-2', 7'-difluorofluorescein (DAF-FM) diacetate were obtained from Invitrogen (Carlsbad, CA, USA).

\section{Animals}

Male Sprague-Dawley rats (8 weeks, 250-300 g) were obtained from the Experimental Animal Center of Xi'an Jiaotong University. Rats were housed in a specific pathogen-free environment under a $12 \mathrm{~h} / 12 \mathrm{~h}$ light/dark cycle. Rats were killed with the inhalation of carbon dioxide. After death was ensured with cervical dislocation, the vessels were collected for later analysis.

\section{Cell culture and treatment}

Bovine aorta endothelial cells (BAECs) were prepared as previously described (Wejksza et al., 2004)) and maintained in DMEM with 10\% FBS, penicillin (100 U.ml ${ }^{-1}$ ) and streptomycin $\left(100 \mathrm{U} \cdot \mathrm{ml}^{-1}\right)$. BAECs were treated with STA $(0-10 \mu \mathrm{M})$ for $24 \mathrm{~h}$ or pre-treated with STA for $12 \mathrm{~h}$ following treatment with Hcy $(500 \mu \mathrm{M})$ for another $12 \mathrm{~h}$. BAECs within seven passages were used.

\section{Determination of cell viability}

Cell viability was evaluated by the MTT assay. BAECs were seeded in 96-well plates and cultured until $80 \%$ confluence. Then, cells were treated with the indicated concentrations of STA for $24 \mathrm{~h}$ before incubation with $5 \mathrm{mg} \cdot \mathrm{ml}^{-1} \mathrm{MTT}$ at $37{ }^{\circ} \mathrm{C}$ in $5 \% \mathrm{CO}_{2}$ atmosphere for $4 \mathrm{~h}$. Next, the culture medium was removed and the formazan formed in the reaction was dissolved in $150 \mu \mathrm{l}$ DMSO. The metabolized MTT was measured by using a spectrophotometer at $490 \mathrm{~nm}$, and calculated by OD (test)/OD (control) $\times 100 \%$.

\section{Nitric oxide (NO) assay}

The levels of NO in the culture supernatants were assayed with the use of the Griess Reagent Nitrite Measurement Kit (Cell Signaling) to detect nitrite, a stable and nonvolatile breakdown product of NO. Briefly, $100 \mu \mathrm{l}$ of supernatant was mixed with $100 \mu \mathrm{l}$ of Griess reagent in a 96-well plate. Nitrite concentration was determined by detecting spectrophotometric absorbance at $550 \mathrm{~nm}$ and plotted against respective concentration in a standard curve $(0-100 \mu \mathrm{M})$ derived from the nitrite standards. Culture media from cell-free wells was used as blank control. Intracellular NO concentrations were measured by using DAF-FM diacetate (4-Amino-5-Methylamino-2',7'-Difluorofluorescein Diacetate). Briefly, BAECs seeded on glass coverslips and treated with different stimuli. By the end of treatment, the cells were incubated with DMEM containing $5 \mu \mathrm{M}$ DAF-FM for $30 \mathrm{~min}$ in the dark at $37^{\circ} \mathrm{C}$ and then washed with PBS. Images were obtained using the fluorescence microscopy. NO production was evaluated by measuring fluorescence intensity.

\section{Measurement of $\mathrm{BH}_{4}$}

$\mathrm{BH} 4$ levels the cell lysates were measured by using a competitive ELISA kit. Briefly, ELISA plates pre-coated with a BH4-specific monoclonal antibody were used to incubate with the samples and test standards along with fixed amount of biotin-labelled BH4. Excess sample and reagents were washed off, and avidin conjugated to HRP was added to each well and incubated again. The 3,3',5,5'-Tetramethylbenzidine (TMB) liquid substrate was then added to each well followed by incubation for $10 \mathrm{~min}$. The enzyme substrate reaction was terminated and the product was measured spectrophotometrically 
at $450 \mathrm{~nm}$. The concentration of $\mathrm{BH} 4$ in each sample was then calculated by comparing the optical density to a standard curve (Almudever et al., 2013).

\section{Measurement of cGMP levels in rat arterial rings}

Rat aortic rings were incubated at $37{ }^{\circ} \mathrm{C}$ in 12 -well culture plates containing Krebs solution gassed with 95\% $\mathrm{O}_{2}$ and $5 \% \mathrm{CO}_{2}$. Tissue lysates were prepared by homogenization with a Polytron homogenizer and centrifugation at $12,000 \times \mathrm{g}$ for $20 \mathrm{~min}$. The levels of cGMP were measured using an enzyme immunoassay kit in accordance to the instructions of the manufacturer.

\section{Measurement of vasorelaxation}

Rat thoracic aortas (TA), mesenteric arteries (MA) and renal arteries (RA) were harvested and immediately transferred into oxygenated ice-cold Krebs solution containing $\mathrm{NaCl}(119 \mathrm{mM}), \mathrm{KCl}(4.7 \mathrm{mM}), \mathrm{NaHCO}_{3}(25 \mathrm{mM}), \mathrm{CaCl}_{2}$ (2.5 mM), $\mathrm{MgCl}_{2}(1 \mathrm{mM}), \mathrm{KH}_{2} \mathrm{PO}_{4}(1.2 \mathrm{mM})$ and $\mathrm{D}-$ glucose $(11 \mathrm{mM})$. In some experiments, the endothelial layer was denuded using $0.1 \%$ Triton X-100. After cleaned perivascular tissues, the arteries were cut into several rings (each in $2 \mathrm{~mm}$ length). Then the rings were suspended between two stainless steel hooks in a $5 \mathrm{~mL}$ organ bath filled with Krebs solution oxygenated with 95\% $\mathrm{O}_{2}-5 \% \mathrm{CO}_{2}$ and maintained at $37{ }^{\circ} \mathrm{C}(\mathrm{pH}$ 7.4) to achieve an optimal tension (TA $10 \mathrm{mN}, \mathrm{RA}$ and MA $3 \mathrm{mN}$ ). Each segment was allowed to equilibrate for $60 \mathrm{~min}$. Curve recording was performed with LabChart ${ }^{\mathrm{tm}}$ software (AD-Instruments, Shanghai, China) by using a PowerLab Data Acquisition System $^{\text {ru }}$ for data acquisition. After equilibration, segments were pre-contracted with $60 \mathrm{mM} \mathrm{KCl}$ twice, and then contracted with phenylephrine $(10 \mu \mathrm{M})$. Once a sustained tension was reached, acetylcholine (ACh, 0.01$10 \mu \mathrm{M})$ was added cumulatively to evoke endotheliumdependent relaxations. TAs with intact endothelia was incubated for $30 \mathrm{~min}$ with each of the following inhibitors: $100 \mu \mathrm{M}$ L-NAME (an NOS inhibitor), $1 \mu \mathrm{M}$ indomethacin (a non-selective COX inhibitor), $100 \mathrm{nM} 1400 \mathrm{~W}$ (an iNOS inhibitor) and $3 \mu \mathrm{M}$ ODQ before the contraction with phenylephrine. In organ culture experiments, aortic rings were incubated in DMEM/F12 supplemented with $10 \%$ FBS oxygenated with $95 \% \mathrm{O}_{2}$ and $5 \% \mathrm{CO}_{2}$ and maintained at $37^{\circ} \mathrm{C}$. After pre-incubation with $10 \mu \mathrm{M}$ STA or vehicle for $12 \mathrm{~h}$, aortic rings were mounted in Myograph system to measure the ACh-induced relaxations.

\section{Quantitative reverse transcriptase-PCR (qRT-PCR)}

Total RNA was isolated using TRIzol (Invitrogen, Carlsbad, CA), reverse transcribed into cDNA by using iScript cDNA synthesis kit (Bio-rad, Hercules, CA). Real-time PCR was performed by using SYBR Green Supermixes (Bio-rad) and a 7500 Real-time PCR machine (Applied Biosystems, Foster City, CA). Fold changes of gene expression were calculated using the $2^{-\Delta \Delta C t}$ method. The qRT-PCR primers used were as follows: GTPCH1 forward primer: 5'-TTGGAAAGGTC CATATCGGT-3', reverse primer: 5'-ATTGTGCTCGTCA CGGTTCT-3'; DHFR forward primer: 5'-AAGAACGG AAACCTGCCCTG-3', reverse primer: 5'-GCCTCCCACT ATCCAAACCA-3'; Nrf2 forward primer: 5'-AGC AC ACCCAGTCAGAAACCAG-3'; reverse primer: 5'-TCTAC AAACGGGAATGTCG-3'; $\beta$-actin forward primer: $5^{\prime}$ CGAGCATTCCCAAAGTTCTACAGTG-3', reverse primer: $5^{\prime}$ - CTACATACTTCCGAAAACCAGGGG-3'. $\beta$-actin was used as an internal control.

\section{Western blotting}

Whole protein samples were extracted with lysis buffer (50 mM Tris- $\mathrm{HCl}, \mathrm{pH}$ 7.5, $15 \mathrm{mM}$ EGTA, $100 \mathrm{mM}$ $\mathrm{NaCl}, 0.1 \%$ Triton $\mathrm{X}-100$ and the protease inhibitors). Cytoplasmic protein samples were extracted with hypotonic lysis buffer $(10 \mathrm{mM}$ Tris- $\mathrm{HCl}, \mathrm{pH} 7.5,1.5 \mathrm{mM}$ $\mathrm{MgCl}_{2}, 10 \mathrm{mM} \mathrm{KCl}$, and $0.5 \% \mathrm{NP} 40$ ). Nuclear protein samples were extracted with high-salt buffer $(20 \mathrm{mM}$ Tris- $\mathrm{HCl}, 1.5 \mathrm{mM} \mathrm{MgCl}, 420 \mathrm{mM} \mathrm{NaCl}, 10 \%$ glycerol, and $0.2 \mathrm{mM}$ EGTA). And then separated on 10\% SDSPAGE and blotted onto PVDF membranes. The blots were incubated with primary antibodies and HRP-conjugated secondary antibodies, and then visualized by using the ECL chemoluminescence system. The intensity of the bands was quantified by using Image Pro Plus software. The eNOS dimerizations were assayed by using lowtemperature SDS-PAGE (LT-PAGE) as described previously (Klatt et al., 1995). The protein lysates were mixed with loading buffer (without $\beta$-mercaptoethanol) and loaded on gels without boiling. Electrophoresis and gel transferring were kept at $4{ }^{\circ} \mathrm{C}$ during the whole procedure.

\section{Small interfering RNA and transfection}

For small interfering RNA (siRNA)-mediated gene knockdown, the siRNA targeting the human Nrf2 gene was synthesized: sense: 5'-GCCCAUUGAUGUUUCUG AUTT-3', antisense: 5'-AUCAGAAACAUCAAUGGGC TT-3'. The siRNAs were transfected into cells using Lipofectamine2000 (Invitrogen). A scrambled siRNA was used as negative control (NC).

\section{Statistical analysis}

Quantitative data are expressed as mean \pm SEM, and data analyses were carried out independently by the third party without knowledge of treatments. Student's $t$ test or ANOVA was used to analyze the differences between two or among more groups, respectively. Relaxation curves were analyzed by using two-way ANOVA followed by Bonferroni post-tests. Bonferroni post hoc tests were run when $\mathrm{F}$ achieved $\mathrm{P}<0.05$ and there was no significant inhomogeneity. Non-quantitative 
results were representative of at least five independent experiments.

\section{Results}

\section{STA improved Hcy-impaired vascular relaxation}

In order to examine the effect of Hcy on vasorelaxation, rat TA, MA and RA rings were incubated with Hcy $(500 \mu \mathrm{M})$ or control medium for $1 \mathrm{~h}$ before measuring the ACh-induced endothelium-dependent relaxation. The effects of STA were assessed by the pre-incubation of artery rings with STA $(10 \mu \mathrm{M})$ for $12 \mathrm{~h}$ before the exposure to Hcy. As shown in Fig. 1, Hcy significantly impaired the vasorelaxations in response to $\mathrm{ACh}$ in all three types of arteries. Clearly, STA effectively restored the vascular relaxation to $\mathrm{ACh}$. Figure $1 \mathrm{~b}-\mathrm{f}$ are representative traces of endothelium-dependent relaxations as in A, C and E. In uninjured rat TA, MA and RA, STA only slightly affected the vasorelaxation (Additional file 1: Figure S1A-C). Taken together, these results suggest that STA protected endothelial function impaired by Hcy.

In addition, we assessed the effects of STA on endothelial dysfunction induced by Ang II and PA, the two common injurious factors associated with metabolic disorders such as hypertension and obesity. in In rat thoracic aortas, exposure to either Ang II $(1 \mu \mathrm{M}, 1 \mathrm{~h})$ or PA $(200 \mu \mathrm{M}, 1 \mathrm{~h})$ significantly impaired vasorelaxations (Additional file 2: Figure S2A and B). However, pretreatment with STA $(10 \mu \mathrm{M}, 12 \mathrm{~h})$ effectively protected the vascular relaxation against Ang II and PA.

STA-improved relaxation was dependent on endotheliumderived NO

To define the contribution of NO in the STA-improved relaxation, the artery rings were treated with different pharmacological inhibitors before the measurement of relaxation. As shown in Fig. 2, either L-NAME (an inhibitor of NOS) or ODQ (an inhibitor of the NO-sensitive soluble guanylyl cyclase, sGC) abolished the effect of STA on the relaxation of endothelium-intact rat thoracic arterial rings. In contrast, neither indomethacin (a non-selective COX inhibitor) nor $1400 \mathrm{~W}$ (an iNOS inhibitor) affect the STA effect (Fig. 2). These results indicated that protective effect of STA was dependent on NO signaling.

\section{STA increased NO, cGMP and BH4 production}

Thus, we examined the effect of STA on NO production in ECs. In cultured BAECs, treatment with STA (0$20 \mu \mathrm{M}$ for $24 \mathrm{~h}$ ) had no significant effect on cell viability, as measured with the MTT assay (Fig. 3a). The production of NO was significantly reduced in BAECs after the exposure to Hcy $(500 \mu \mathrm{M}$ for $12 \mathrm{~h})$. However, pretreatment with STA $(10 \mu \mathrm{M}, 12 \mathrm{~h})$ effectively attenuated the detrimental effect of Hcy on NO production (Fig. 3b). The effect of STA on NO production was confirmed with laser confocal microscopy using a specific fluorescence dye

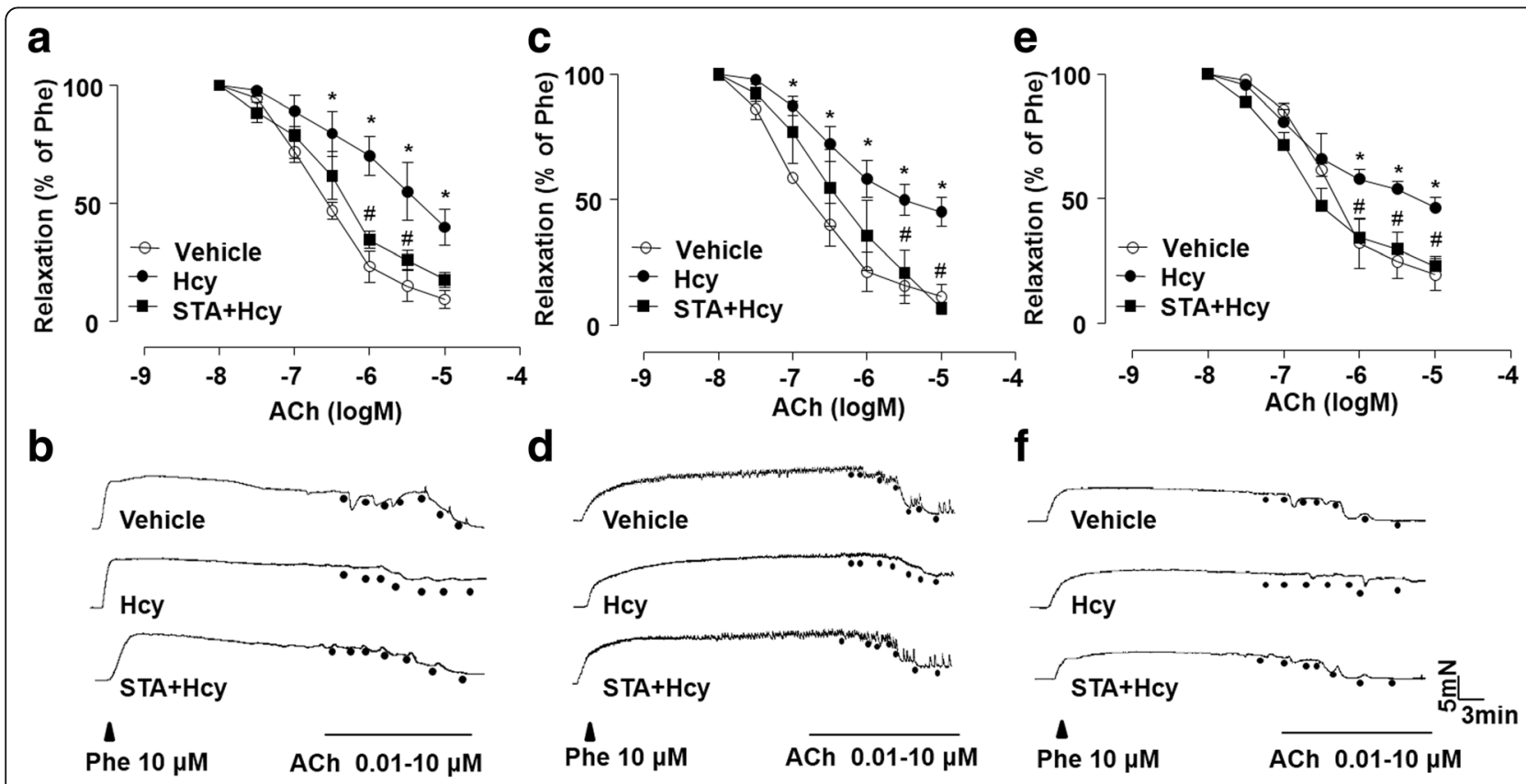

Fig. 1 STA improved Hcy-impaired vascular relaxation. After pre-treatment of TA (a), MA (c) and RA (e) rings with STA (10 $\mu$ M, 12 h), the arterial rings were exposed to Hcy (500 $\mu \mathrm{M}, 60 \mathrm{~min})$. The cumulative concentration-response curves for ACh-induced relaxations of the phenylephrine-pre-contracted arterial rings were shown. $\mathbf{b}, \mathbf{d}$ and $\mathbf{f}$ Representative traces of endothelium-dependent relaxations as in (a, $\mathbf{c}$ and $\mathbf{e})$. Data were shown as mean $\pm S E M ; n=5$ for each group, ${ }^{*} P<0.05$ vs. Vehicle; ${ }^{\#} P<0.05$ vs. Hcy 


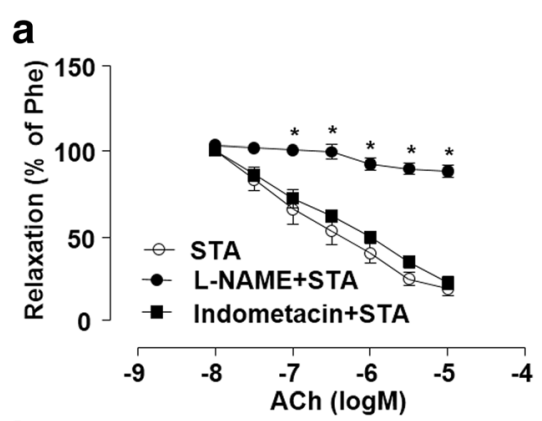

b

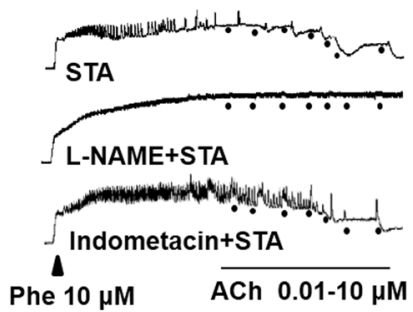

C

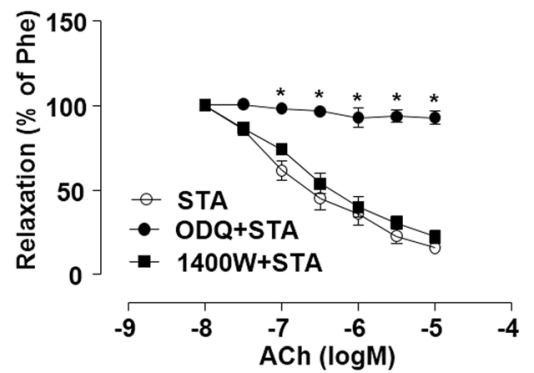

d

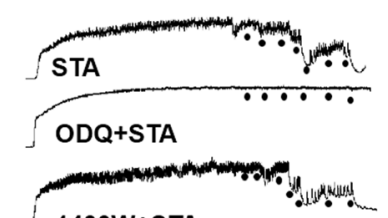

$1400 \mathrm{~W}+\mathrm{STA}$

Phe $10 \mu \mathrm{M}$

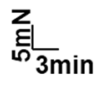

Fig. 2 STA-improved relaxation was dependent on endothelium-derived NO. Rat thoracic artery rings were incubated with STA (10 $\mu M)$ for $12 \mathrm{~h}$ and exposed to L-NAME $(100 \mu \mathrm{M})$, indomethacin $(1 \mu \mathrm{M})(\mathbf{a}), 1400 \mathrm{~W}(100 \mathrm{nM})$ or ODQ $(3 \mu \mathrm{M})(\mathbf{c})$ for 30 min before the PE-contraction and AChinduced relaxation. $\mathbf{b}$ and $\mathbf{d}$ Representative traces of endothelium-dependent relaxations as in (a and $\mathbf{c}$ ). Data were shown as mean $\pm \mathrm{SEM} ; n=5$, ${ }^{*} P<0.05$ vs. Vehicle

DAF-FM (Fig. 3c and d). Since endothelium-derived NO acts on arterial walls to promotes vasorelaxation by producing cGMP via the activation of sGC (Papapetropoulos et al., 2015), we further determined the cGMP levels in arterial rings treated with STA. As shown in Fig. 3e, The Hcy-decreased cGMP production was prevented by STA. $\mathrm{BH} 4$ is an essential cofactor for eNOS to completely couple NADPH oxidation to NO production. As shown in Fig. 3f, treatment with Hcy ( $500 \mu \mathrm{M}, 12$ h) significantly reduced $\mathrm{BH} 4$ production compared with vehicle control. However, pretreatment with STA effectively restored the BH4 level in BAECs. These results suggest that increase of $\mathrm{BH} 4$ and the ensuing activation of NO pathway may contribute to the protective effect of STA.

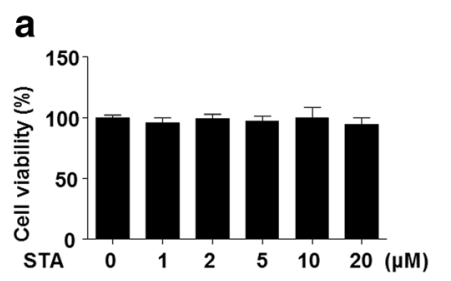

e

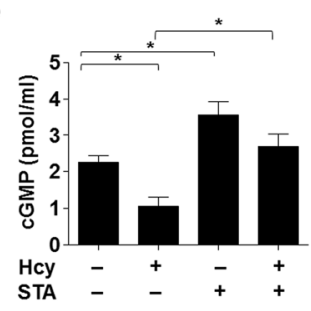

b

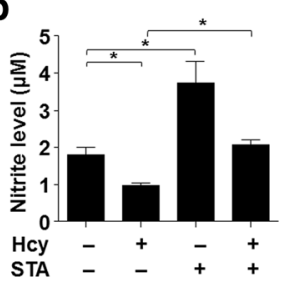

f

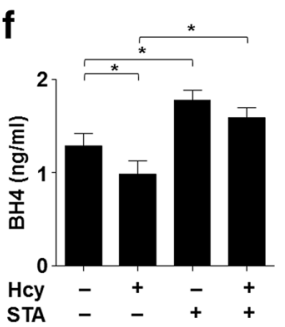

C

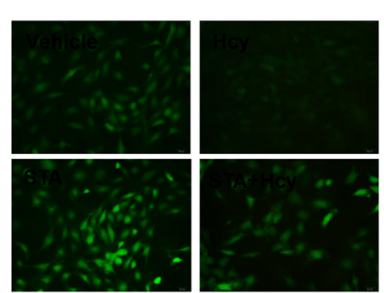

d

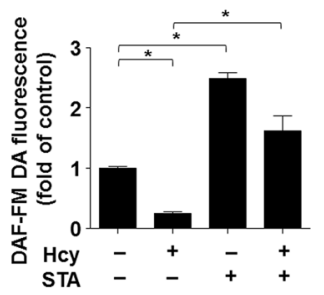

Fig. 3 STA increased NO, CGMP and BH4 production. a BAECs were treated with indicated concentrations of STA for 24 h, and cell viability was measured by using MTT. b BAECs were pre-incubated with STA (10 $\mu \mathrm{M})$ or vehicle for $12 \mathrm{~h}$, and then exposed to Hcy (500 $\mu \mathrm{M}$, $12 \mathrm{~h}$ ). NO concentration in supernatants was measured by using the Griess reagent. c Intracellular NO was detected by using DAF-FM probe (40 x objective). d The mean fluorescence intensity was evaluated as in (c). e Rat thoracic artery rings pre-incubated with STA or vehicle were exposed to Hcy. The cGMP production was measured by using an enzyme immunoassay kit. $\mathbf{f}$ Intracellular levels of BH4 were measured by using BH4 ELISA Kit. Data shown were mean \pm SEM, $n=5,{ }^{*} P<0.05$ 
STA ameliorated Hcy-induced eNOS uncoupling

It is established that $\mathrm{BH} 4$ plays an important role in facilitating eNOS dimerization, which is necessary for its normal catalytic function. Thus, we investigated the effects of STA on eNOS dimerization. As shown in Fig. $4 \mathrm{a}$ and $\mathrm{b}$, the ratio of eNOS dimers to monomers, assayed by low-temperature SDS-PAGE, was significantly decreased in Hcy-treated ECs. However, pretreatment with STA ameliorated Hcy-decreased ratio of eNOS dimer to monomer. In fact, we observed that STA increased the dimer/monomer ratio in a dose-dependent manner (Fig. 4c and d). These results suggest that STA attenuated Hcy-induced eNOS uncoupling.

\section{STA increased the levels of GTPCH1 and DHFR}

GTPCH1 and DHFR are the two key catalyzing the biosynthesis of BH4 and NO. Therefore, we further assessed the effect of STA on the expression of these two genes in ECs by using qRT-PCR and Western blotting. As shown in Fig. 5a-c, Hcy reduced the mRNA and protein levels of GTPCH1 and DHFR, which were significantly reversed by STA pretreatment. Furthermore, STA increased mRNA and protein levels of GTPCH1 and DHFR in dose- and time-dependent manners (Fig. 5d-h). These results suggested that induction of GTPCH1 and DHFR may contribute to the protection against the Hcy-impaired $\mathrm{BH} 4$ and $\mathrm{NO}$ production.

\section{STA increased the expressions of GTPCH1 and DHFR via activation of Nrf2}

Nuclear factor erythroid 2-related factor 2 (Nrf2) transcriptionally controls the gene expression of many cytoprotective enzymes, such as heme oxygenase-1 (HO-1)
(Swamy et al., 2016) and NAD(P)H:quinone reductase (NQO1) (Rohrer et al., 2014). Particularly, it was shown that Nrf2 regulated-GTPCH1/BH4 axis ameliorated skin injury (Xue et al., 2017). To test whether Nrf2 is involved in the effects of STA on the induction of expressions of GTPCH1 and DHFR, BAECs were treated with STA $(10 \mu \mathrm{M})$ for different time periods and the Nrf2 levels were detected by using western blotting. As shown in Fig. 6a, STA increased the total Nrf2 protein level. Furthermore, STA treatment rapidly increased Nrf2 protein level in the nuclear portions (Fig. $6 \mathrm{~b}$ and c), suggesting that Nrf2 is activated and likely translocated into the nuclear compartment in response to STA exposure. Furthermore, Nrf2 knockdown by siRNA abrogated STAincreased levels of GTPCH1 and DHFR (Fig. 6d and e). These results indicated that activation of Nrf2 might act as a transcriptional regulator to mediate the STAinduced gene expression of GTPCH1 and DHFR.

\section{Discussion}

Endothelial dysfunction has been implicated in the pathogenesis of CVDs such as atherosclerosis, diabetes and hypertension (Martin-Timon et al., 2014). STA is belongs to naturally occurring alkaloids. Among their diverse pharmacological properties such as anti-microbial, anti-tumor, anti-diabetic and anti-inflammatory effects, many natural alkaloids have been reported to possess vascular protective activities (Kittakoop et al., 2014; Cushnie et al., 2014). However, the molecular mechanisms underlying such vascular benefit remain largely unknown. In the present study, we for the first time provided both in vitro and ex vivo evidence that STA protects against the Hcy-impaired endothelial dysfunction.
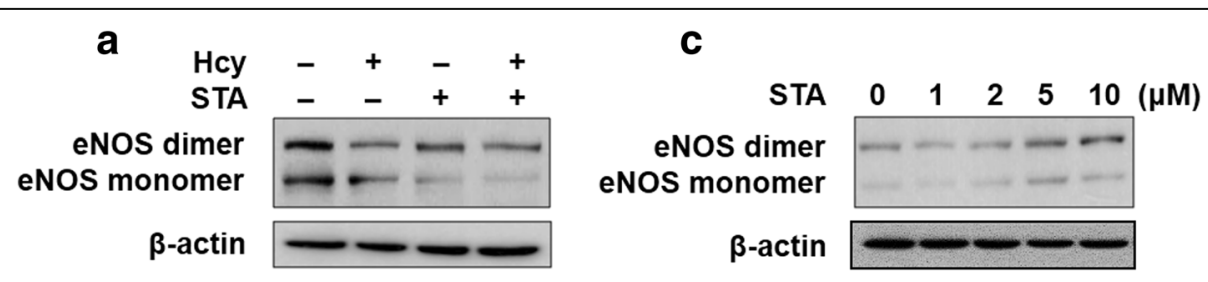

b

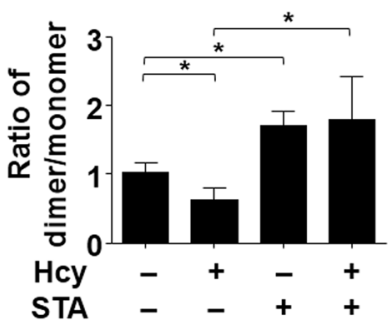

d

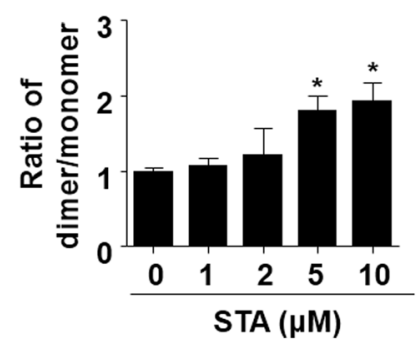

Fig. 4 STA ameliorated Hcy-induced eNOS uncoupling. a BAECs were pre-incubated with STA (10 $\mu \mathrm{M}, 12 \mathrm{~h})$ or vehicle and, then exposed to Hcy $(500 \mu \mathrm{M}, 12 \mathrm{~h})$. Protein levels of eNOS dimers and monomers were detected by using low-temperature SDS-PAGE/western blotting. c BAECs were exposed to indicated concentrations of STA for $24 \mathrm{~h}$, cell lysates were analyzed to determine eNOS dimers and monomers protein levels. $\mathbf{b}$ and $\mathbf{d}$ Quantification of eNOS dimer/monomer levels. Data were shown as mean \pm SEM, $n=5 .{ }^{*} P<0.05$ 


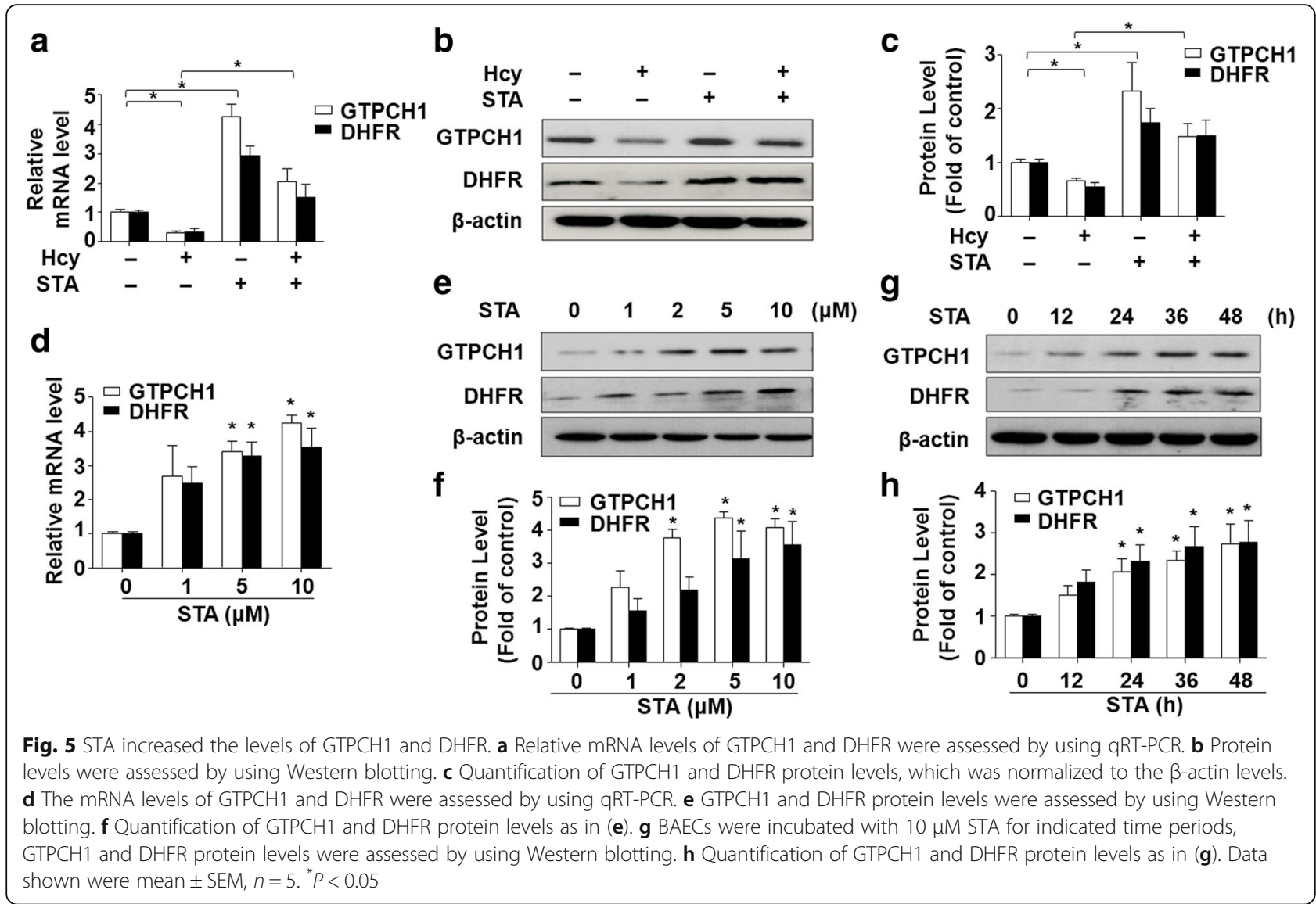

Most importantly, we defined the novel mechanisms by which STA enhances eNOS coupling and NO bioavailability via up-regulation of GTPCH1 and DHFR, the genes for two major enzymes responsible for the de novo biosynthesis and salvage pathways of $\mathrm{BH} 4$. Given the critical roles of NO signaling in the vascular homeostasis and diseases, these findings are of pathophysiological importance to our understanding of the pharmacological mechanisms by which the naturally occurring compounds exert their vascular actions.

Hcy is an intermediate metabolite in the metabolic pathway of cysteine and methionine (Gurda et al., 2015). Elevated level of plasma Hcy is an independent risk factor of CVD and Hcy contributes to the development of CVD as it causing endothelial dysfunction (Ivanov et al., 2015). In our study, we found that STA prevented Hcyinduced impairment of endothelium-dependent relaxation (Fig. 1). L-NAME, a NOS inhibitor, suppressed the relaxation of STA, indicating that the vasorelaxant effect of STA was associated with NO release. This notion is corroborated with the result that ODQ, a NO-sensitive guanylyl cyclase inhibitor that blocks the signaling pathway of the endothelium-derived NO, also reduced STAinduced relaxation. By contrast, iNOS did not play any roles in the enhanced NO release, which was confirmed using the iNOS inhibitor $1400 \mathrm{~W}$ (Fig. 2). These results clearly demonstrated that STA can elicit a NO-dependent vasodilation.

ECs produce a variety of vasoactive substances, such as $\mathrm{NO}$, endothelium-derived hyperpolarizing factor (EDHF), prostacyclin, or endothelin-1 (ET-1), Ang II, thromboxane A2 and prostaglandin $\mathrm{H} 2$, to keep the delicate balance between vasodilation and vasoconstriction (Husain et al., 2015). Among these endothelial-derived vasodilators, NO is the most important one because of its key role in inhibiting platelet aggregation, inflammation, oxidative stress, vascular smooth muscle cell migration and proliferation, and leukocyte adhesion (Zhao et al., 2015). In our study, we showed that the impaired production of $\mathrm{NO}$ by Hcy was rescued by STA (Fig. 3). NO-induced relaxation is associated with increased levels of cGMP in vascular smooth muscle cells. An elevation in intracellular cyclic GMP levels leads to vasorelaxation. Our findings indicated that the effect of STA-induced relaxation via NO-dependent cGMP production. eNOS catalyzes the biosynthesis of NO in endothelial cells whereas $\mathrm{BH} 4$ is a critical determinant of eNOS activity. We demonstrated that STA prevented the Hcy-induced $\mathrm{BH} 4$ decrease and that STA increased $\mathrm{BH} 4$ production in ECs (Fig. 3). BH4 bioavailability in the vasculature appears to be regulated by both a de 

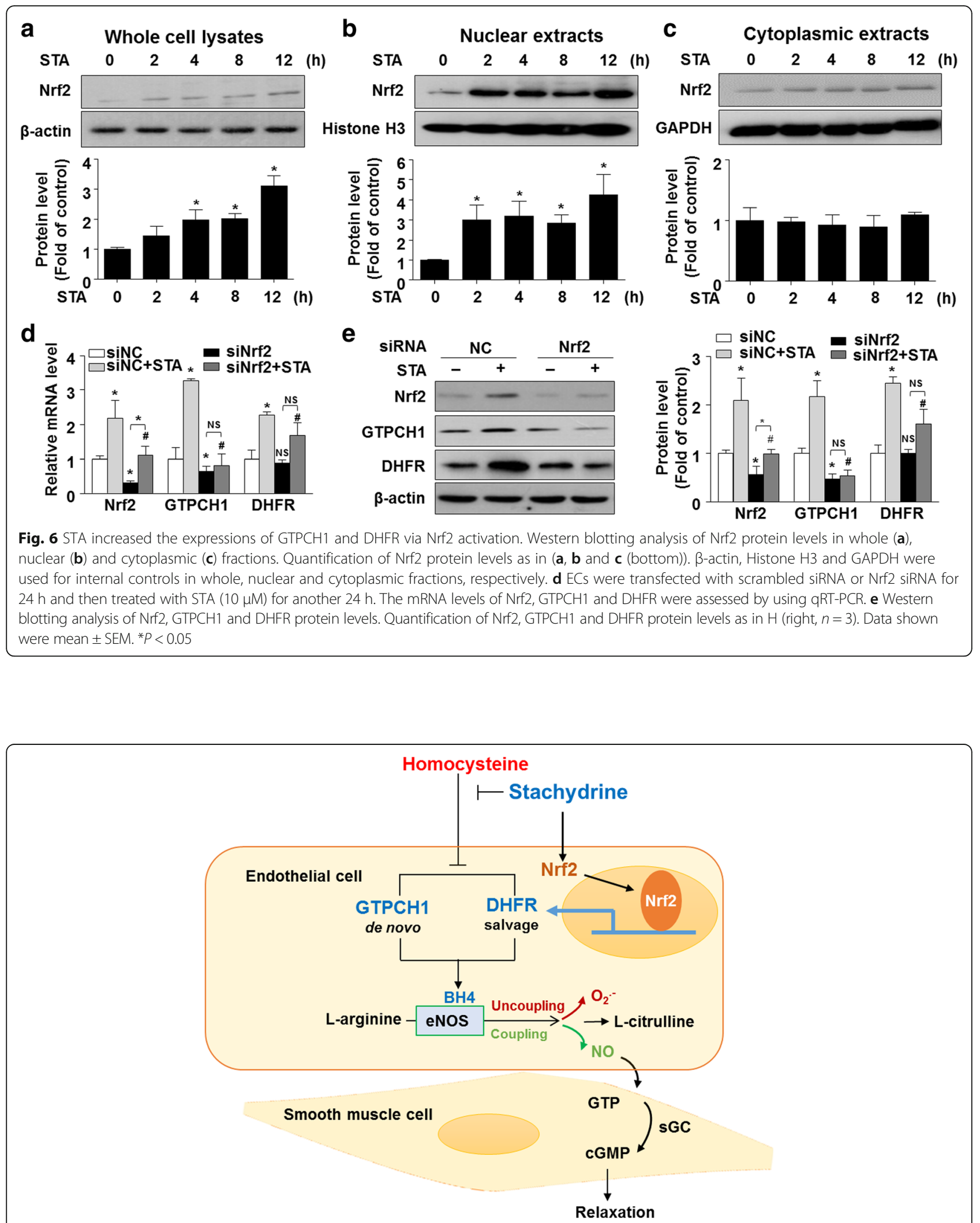

Fig. 7 Schematic model of the effect of STA on Hcy-induced endothelial dysfunction. Stachydrine ameliorated Hcy-induced endothelial dysfunction via Nrf2-dependent up-regulation of GTPCH1 and DHFR. The de novo synthesis and salvage of BH4 reversed eNOS uncoupling and increased NO production, thus, leading to improved endothelium-dependent vasorelaxation 
novo pathway using the rate-limiting enzyme GTPcyclohydrolase I (GTPCH1) and a salvage pathway from the synthetic pterin, sepiapterin, which is metabolized to $\mathrm{BH} 4$ by sepiapterin reductase (SR) and endothelial dihydrofolate reductase (DHFR) (Bendall et al., 2014). It has been previously demonstrated that depletion of $\mathrm{BH} 4$ renders uncoupling eNOS uncoupled from L-arginine oxidation, resulting in generation of $\mathrm{O}^{-}$rather than NO. Such an "eNOS uncoupling" status is thought to contribute to vascular oxidative stress and endothelial dysfunction (Hsieh et al., 2014). In fact, it was previously described that homocysteine exposure could lead to eNOS uncoupling in EC (Topal et al., 2004). The mechanisms underlying the suppressive effects of NO bioavailability and eNOS uncoupling are still incompletely understood. In light of the previous findings that Hcy significantly reduced intracellular BH4 level in ECs and that ascorbic acid, a BH4 regenerator, rescued the NO production in Hcy-exposed ECs (Topal et al., 2004), a decreased in BH4 level or, more importantly, the BH4:BH2 ratio (Crabtree et al., 2009), may account for the deleterious effect of homocysteine. Interestingly, we found STA markedly increased eNOS coupling, as suggested by the increased levels of NO production, dimerized eNOS, $\mathrm{BH} 4: \mathrm{BH} 2$ ratio, as well as GTPCH1 and DHFR expression in ECs (Figs. 4 and 5).

$\mathrm{Nrf} 2$ is sequestered in the cytoplasmic portion and bind to its repressor molecule, Keap1 (Soares et al., 2016). Under stressful conditions such as oxidative or ER stress, Nrf2 dissociates from the Nrf2-Keap1 complex. Subsequently, Nrf2 undergoes nuclear translocation to transcriptionally activate its target genes. In the present study, Nrf2 was rapidly increased by STA in ECs. Nrf2 was increased in the nuclear portion within $2 \mathrm{~h}$ after the STA treatment, indicating that Nrf2 activation is an early event in the STA signaling. A critical role of Nrf2 was further supported by the results that gene silencing of Nrf2 attenuated the STA induction of both GTPCH1 and DHFR (Fig. 6). Given that both GTPCH1 and DHFR genes harbor the cognate cis-elements within the 5 '-flanking regions, we speculate that STA may activate Nrf2 to transcriptionally up-regulate the gene expression of GTPCH1 and DHFR, which are responsible for the de novo biosynthesis and salvage pathways of $\mathrm{BH} 4$; such a coordinated gene induction result in increased $\mathrm{BH} 4$ and $\mathrm{NO}$ bioavailability, thus protecting endothelial-dependent vascular function against such metabolic risk factor as homocysteinemia, saturated free fatty acids and Ang II (Fig. 7).

\section{Conclusion}

Taken together, our results demonstrated that stachydrine ameliorated Hcy-induced endothelial dysfunction via Nrf2 dependent up-regulation of GTPCH1 and DHFR and increase in bioavailabilities of $\mathrm{BH} 4$ and $\mathrm{NO}$. These results revealed a new mechanism by which the natural alkaloids
STA protect endothelial function in response to such risk factors as increased concentration of homocysteine.

\section{Additional files}

Additional file 1: Figure S1. STA slightly improved the vasorelaxation in rat TA, MA, and RA. (TIFF $289 \mathrm{~kb}$ )

Additional file 2: Figure S2. STA improved Ang II or PA-impaired vascular relaxation. (TIFF $258 \mathrm{~kb}$ )

\section{Abbreviations}

BH4: Tetrahydrobiopterin; CVDs: Cardiovascular diseases; DHFR: Dihydrofolate reductase; ECs: Endothelial cells; eNOS: Endothelial nitric oxide synthase; GTPCH1: Guanosine triphosphate cyclohydrolase-1; Hcy: Homocysteine; LNAME: NG-nitro-L-arginine methylester; MA: Mesenteric arteries; NO: Nitric oxide; Nrf2: Nuclear factor erythroid 2-related factor 2; ODQ: 1H- [1, 2, 4] Oxadiazolo [4,3-a] quinoxalin-1-one; RA: Renal arteries; STA: Stachydrine; TA: Thoracic aortas

\section{Acknowledgements}

We would like to thank Yahan Liu and Lei Qian for helpful discussion.

\section{Funding}

This study was supported by grants from the National Science Foundation of China (31430045, 81770497, 81470373 and 81220108005).

\section{Availability of data and materials}

The datasets used and/or analysed during the current study are available from the corresponding author on reasonable request. All data generated or analysed during this study are included in this published article [and its Additional files].

\section{Authors' contributions}

XX performed most of experiments, analyzed the data and wrote the draft of the manuscript. ZZ, XW and ZL performed a part of experiments. BL contributed new reagents or analytic tools. LX and NW designed the experiments, provided funding, and wrote the manuscript. All authors read and approved the final manuscript.

\section{Ethics approval}

The protocols for the animal experiments were approved by the institutional review board of Xi'an Jiaotong University and performed in accordance with the institutional and national guidelines for the care and use of animals (No. XJTULAC 2015-646).

\section{Consent for publication}

Not applicable.

\section{Competing interests}

The authors declare that they have no competing interests as defined by Molecular Medicine or other interests that might be perceived to influence the results and discussion reported in this paper.

\section{Publisher's Note}

Springer Nature remains neutral with regard to jurisdictional claims in published maps and institutional affiliations.

Received: 21 February 2018 Accepted: 2 March 2018

Published online: 19 March 2018

\section{References}

Almudever $\mathrm{P}$, et al. Role of tetrahydrobiopterin in pulmonary vascular remodelling associated with pulmonary fibrosis. Thorax. 2013;68:938-48.

Baggott JE, Tamura T. Homocysteine, iron and cardiovascular disease: a hypothesis. Nutrients. 2015;7:1108-18.

Bendall JK, Douglas G, McNeill E, Channon KM, Crabtree MJ. Tetrahydrobiopterin in cardiovascular health and disease. Antioxid Redox Signal. 2014;20:3040-77.

Cheng Z, et al. Hyperhomocysteinemia and hyperglycemia induce and potentiate endothelial dysfunction via mu-calpain activation. Diabetes. 2015;64:947-59. 
Crabtree MJ, et al. Quantitative regulation of intracellular endothelial nitric-oxide synthase (eNOS) coupling by both tetrahydrobiopterin-eNOS stoichiometry and biopterin redox status: insights from cells with tet-regulated GTP cyclohydrolase I expression. J Biol Chem. 2009;284:1136-44.

Cushnie TP, Cushnie B, Lamb AJ. Alkaloids: an overview of their antibacterial, antibiotic-enhancing and antivirulence activities. Int J Antimicrob Agents. 2014;44:377-86.

Gurda D, Handschuh L, Kotkowiak W, Jakubowski H. Homocysteine thiolactone and $\mathrm{N}$-homocysteinylated protein induce pro-atherogenic changes in gene expression in human vascular endothelial cells. Amino Acids. 2015; 47:1319-39.

Haruki H, Hovius R, Pedersen MG, Johnsson K. Tetrahydrobiopterin biosynthesis as a potential target of the kynurenine pathway metabolite Xanthurenic acid. J Biol Chem. 2016;291:652-7.

Hsieh HJ, Liu CA, Huang B, Tseng AH, Wang DL. Shear-induced endothelial mechanotransduction: the interplay between reactive oxygen species (ROS) and nitric oxide (NO) and the pathophysiological implications. J Biomed Sci. 2014;21:3.

$\mathrm{Hu}$ Y, He K, Zhu H. Chinese herbal medicinal ingredients affect secretion of NO, IL-10, ICAM-1 and IL-2 by endothelial cells. Immunopharmacol Immunotoxicol. 2015:37:324-8.

Hu YY, He KW, Guo RL. Six alkaloids inhibit secretion of IL-1alpha, TXB(2), ET-1 and Eselectin in LPS-induced endothelial cells. Immunol Investig. 2012;41:261-74.

Husain K, Hernandez W, Ansari RA, Ferder L. Inflammation, oxidative stress and renin angiotensin system in atherosclerosis. World J Biol Chem. 2015;6:209-17.

Hussein D, et al. Validating the GTP-cyclohydrolase 1-feedback regulatory complex as a therapeutic target using biophysical and in vivo approaches. Br J Pharmacol. 2015;172:4146-57.

Ivanov AN, Puchinyan DM, Norkin IA. Vascular endothelial barrier function. Usp Fiziol Nauk. 2015;46:72-96.

Karolczak K, Kamysz W, Karafova A, Drzewoski J, Watala C. Homocysteine is a novel risk factor for suboptimal response of blood platelets to acetylsalicylic acid in coronary artery disease: a randomized multicenter study. Pharmacol Res. 2013;74:7-22

Kittakoop P, Mahidol C, Ruchirawat S. Alkaloids as important scaffolds in therapeutic drugs for the treatments of cancer, tuberculosis, and smoking cessation. Curr Top Med Chem. 2014;14:239-52.

Klatt P, Schmidt K, Lehner D, Glatter O, Bachinger HP, Mayer B. Structural analysis of porcine brain nitric oxide synthase reveals a role for tetrahydrobiopterin and Larginine in the formation of an SDS-resistant dimer. EMBO J. 1995;14:3687-95.

Kuchta K, Volk RB, Rauwald HW. Stachydrine in Leonurus cardiaca, Leonurus japonicus, Leonotis leonurus: detection and quantification by instrumental HPTLC and 1H-qNMR analyses. Die Pharmazie. 2013;68:534

Martin-Timon I, Sevillano-Collantes C, Segura-Galindo A, Del CF. Type 2 diabetes and cardiovascular disease: have all risk factors the same strength? World J Diabetes. 2014;5:444-70.

Papapetropoulos A, Hobbs AJ, Topouzis S. Extending the translational potential of targeting NO/CGMP-regulated pathways in the CVS. Br J Pharmacol. 2015; 172:1397-414.

Rohrer PR, Rudraiah S, Goedken MJ, Manautou JE. Is nuclear factor erythroid 2-related factor 2 responsible for sex differences in susceptibility to acetaminophen-induced hepatotoxicity in mice? Drug Metab Dispos. 2014:42:1663-74.

Servillo L, et al. Stachydrine ameliorates high-glucose induced endothelial cell senescence and SIRT1 downregulation. J Cell Biochem. 2013;114:2522-30.

Soares MA, et al. Restoration of Nrf2 signaling normalizes the regenerative niche. Diabetes. 2016:65:633-46.

Swamy SM, Rajasekaran NS, Thannickal VJ. Nuclear factor-Erythroid-2-related factor 2 in aging and lung fibrosis. Am J Pathol. 2016;186:1712-23.

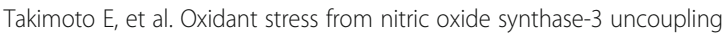
stimulates cardiac pathologic remodeling from chronic pressure load. J Clin Invest. 2005;115:1221-31.

Topal G, et al. Homocysteine induces oxidative stress by uncoupling of NO synthase activity through reduction of tetrahydrobiopterin. Free Radic Biol Med. 2004;36:1532-41.

Wejksza K, et al. Kynurenic acid production in cultured bovine aortic endothelial cells. Homocysteine is a potent inhibitor. Naunyn Schmiedeberg's Arch Pharmacol. 2004:369:300-4.
Xue J, et al. The Nrf2/GCH1/BH4 Axis ameliorates radiation-induced skin injury by modulating the ROS Cascade. J Invest Dermatol. 2017;137:2059-68.

Yin J, et al. Stachydrine, a major constituent of the Chinese herb leonurus heterophyllus sweet, ameliorates human umbilical vein endothelial cells injury induced by anoxia-reoxygenation. Am J Chin Med. 2010;38:157-71.

Zhao Y, Vanhoutte PM, Leung SW. Vascular nitric oxide: Beyond eNOS. J Pharmacol Sci. 2015;129:83-94

\section{Submit your next manuscript to BioMed Central and we will help you at every step:}

- We accept pre-submission inquiries

- Our selector tool helps you to find the most relevant journal

- We provide round the clock customer support

- Convenient online submission

- Thorough peer review

- Inclusion in PubMed and all major indexing services

- Maximum visibility for your research

Submit your manuscript at www.biomedcentral.com/submit
) Biomed Central 